\title{
Pós-graduação em Computação na Universidade de Brasília: Um Grande Desafio na Diversidade de Gênero
}

\author{
Maristela Holanda, Aletéia Patrícia Favacho de Araújo \\ Departamento de Ciência da Computação - Universidade de Brasília (UnB) \\ Campus Darcy Ribeiro - Brasília - DF - Brasil \\ $\{$ mholanda, aleteia\} @unb.br
}

\begin{abstract}
The gender gap in Computer Science is a fact in undergraduate Major in Brazil. However, there are few studies about the gap gender in the master and doctoral degrees. This paper presents the gender gap in the Graduate Program at the University of Brasilia, Brazil. That program has academic master and doctoral degrees, and the professional master degree.

Resumo. O problema de diversidade de gênero em Ciência da Computação é um fato bem documentado em cursos de graduação no Brasil. Quando se trata de pesquisa, mestrado e doutorado, esse diagnóstico é menos conhecido. Assim, este artigo apresenta dados dos programas de pós-graduação da Universidade de Brasília que tem mestrados acadêmico e profissional, e doutorado acadêmico.
\end{abstract}

\section{Introdução}

Ao longo dos anos, o número de mulheres na área de Ciência da Computação diminuiu no Brasil [SBC, 2016][Maia, 2016]. Porém, essa não é uma realidade apenas no Brasil, por exemplo, nos Estados Unidos [Keinan, 2017], Canadá [Chopra et al. 2019], Iugoslávia [Putnik et al. 2017], o número de mulheres em cursos de graduação em Computação tem diminuído a partir dos anos 90s. Na Universidade de Brasília (UnB), o número de meninas nos cursos de graduação em Ciência da Computação, Engenharia da Computação e Licenciatura em Computação é menor que 20\% [Holanda 2017].

Em relação à inserção feminina na pesquisa e na pós-graduação tem-se, segundo dados da UNESCO, que as mulheres estavam entre $44 \%$ e $54 \%$ de todos os formandos, em uma pesquisa realizada em 12 países, dentre esses o Brasil, em 2013. No Japão esta taxa foi de 33\% de mulheres na pós-graduação [Elsevier, 2018]. Entretanto, quando observada a Ciência da Computação e o Brasil, a proporção de mulheres na pós-graduação é bem menor, chegando, por exemplo, a apenas $14 \%$ no nível de doutorado na Universidade de Brasília.

Um outro indicador importante é como está a participação feminina nas publicações científicas. O número de publicação de artigos científicos envolvendo pesquisadoras brasileiras em computação, entre os anos de 2011 e 2015, era em torno de 5.985 artigos, enquanto que os pesquisadores homens participavam de 19.896 artigos [Elsevier 2018]. Assim sendo, as pesquisadoras representaram apenas 23,12\% das publicações no Brasil. 
Neste contexto, este artigo apresenta um estudo sobre gênero em pós-graduação no Departamento de Computação da UnB, uma universidade pública brasileira, em relação à formação de alunas de mestrado e de doutorado, acadêmico e profissional. $\mathrm{O}$ objetivo é responder às seguintes questões de pesquisa: Quando se trata de pesquisa, mestrado e doutorado, como está a formação das mulheres? Em quais áreas da computação estão sendo formadas as futuras doutoras e mestres?

Para isso, este artigo está está dividido, além desta seção, em mais três seções. A Seção 2 apresenta dados da pós-graduação em Computação da UnB. Na Seção 3 uma discussão sobre os resultados são apresentados. Por fim, a Seção 4 apresenta algumas conclusões.

\section{Dados da Pós-graduação em Computação na UnB}

O Departamento de Ciência da Computação da UnB tem três cursos de pós-graduação: mestrado acadêmico, mestrado profissional e doutorado acadêmico. A metodologia aplicada na análise dos dados foi, inicialmente, uma coleta de dados no repositório institucional da UnB [UnB, 2019]. Em seguida, a construção da base de dados, com dados das teses/dissertações tais como, título do trabalho, ano de defesa, nome e sexo do(a) discente, nome e sexo do(a) docente e a subárea da Computação do trabalho. Por fim, uma análise estatística foi aplicada e os resultados são apresentados a seguir.

A Figura 1 apresenta os dados de 280 dissertações e teses defendidas no Departamento de Computação da UnB. O gráfico começa em 2006, ano da primeira publicação disponível no repositório da UnB do departamento. O curso de doutorado iniciou em 2010, e o mestrado profissional em 2012, com isso o aumento no número de defesas a partir do ano de 2014. É possível observar também que há uma enorme diferença entre o número de mulheres e homens que defenderam suas teses e dissertações. Nos anos de 2007, 2008, 2010 e 2012 não houve nenhuma mulher defendendo mestrado ou doutorado na área de Computação nesta instituição.

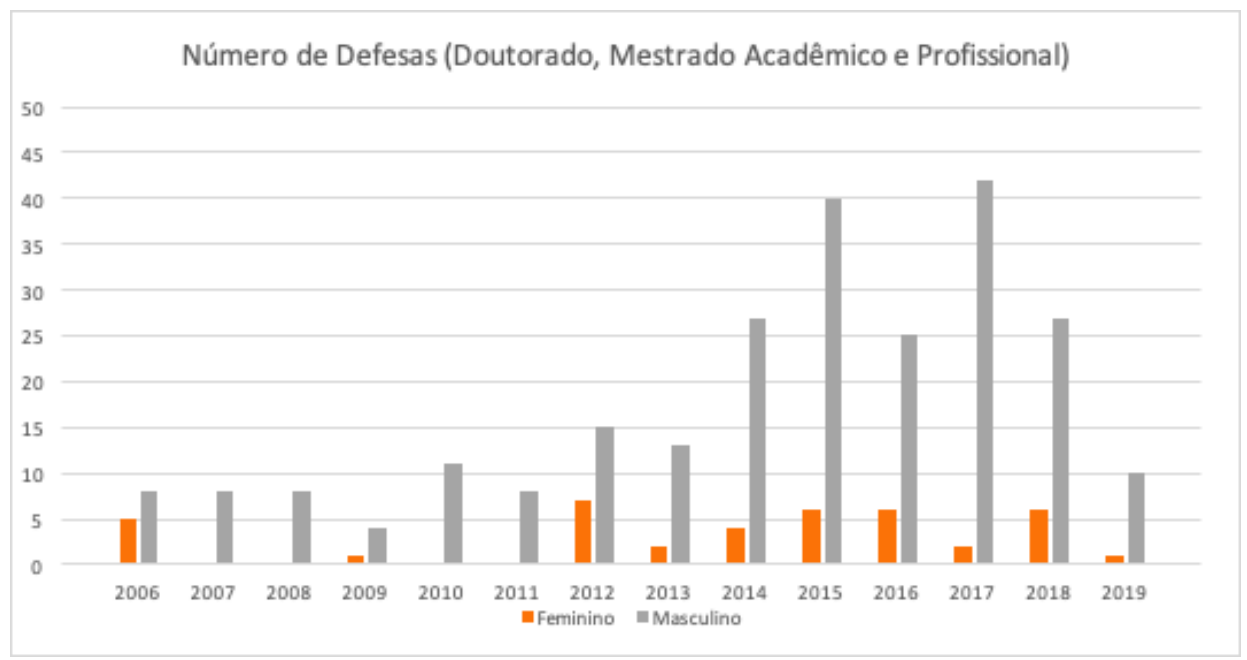

Figure 1. Quantidade de títulos de mestrado acadêmico, mestrado profissional e doutorado acadêmico na área de Computação na UnB. 
A Figura 2 apresenta a proporção de discentes formados nos três programas por sexo. Como é possível observar, o padrão de formação nos programas de mestrado acadêmico e profissional, e doutorado acadêmico segue o mesmo. Nos três níveis menos de $15 \%$ de formação de mulheres. Em termos de números absolutos, das 21 defesas de teses, apenas 3 foram de mulheres; das 163 defesas de dissertações do mestrado acadêmico, apenas 25 são mulheres; e das 103 defesas de dissertações no mestrado profissional apenas 12 são mulheres.
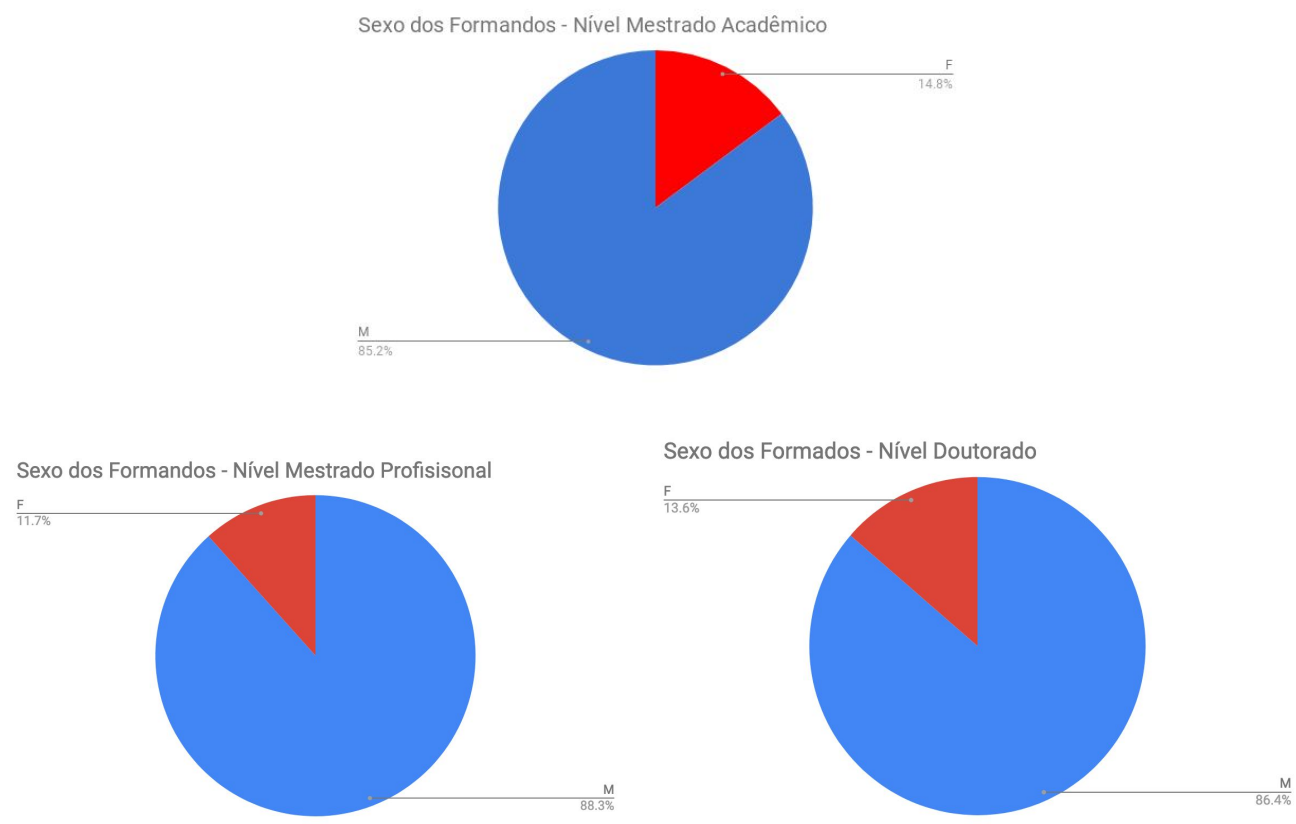

Figura 2. Formação no Mestrado Acadêmico, Mestrado Profissional e Doutorado Acadêmico.

Em relação à área de pesquisa das alunas na pós-graduação em Computação da Universidade de Brasília tem-se: no doutorado, uma aluna é doutora em Bioinformática, uma em Sistema de Informação e a outra em Teoria da Computação. No mestrado acadêmico as áreas são distribuídas como apresentado na Figura 3, sendo que a área de Sistema de Informação teve o maior número de tituladas. O mestrado profissional da UnB é dividido em 4 linhas de pesquisa (Figura 4), sendo Infraestrutura, Ciência dos Dados, Gestão de Risco e Engenharia de Software. Não teve nenhuma aluna na àrea de Infraestrutura titulada no programa profissional, e a área com o maior número de formanda é Gestão Risco, seguido de Ciência dos Dados e Engenharia de Software.

\section{Discussão dos Resultados}

O estudo apresentou uma grande diferença na formação de mestres e doutores em Computação quando observada a questão de gênero. Na área de Infraestrutura a inserção feminina é mínima, em sete anos do programa profissional nenhuma mulher obteve o título nesta área, na UnB.

Assim, notou-se que aumentar o número de mulheres em nível de pós-graduação na Computação é também um grande desafio. Como é conhecido, tem-se o número 
restrito de meninas com graduação em Computação no Brasil, na pós-graduação, que é o topo da pirâmide de ensino, este problema aparece mais fortemente.

\section{Defesas por Área de Conhecimento}

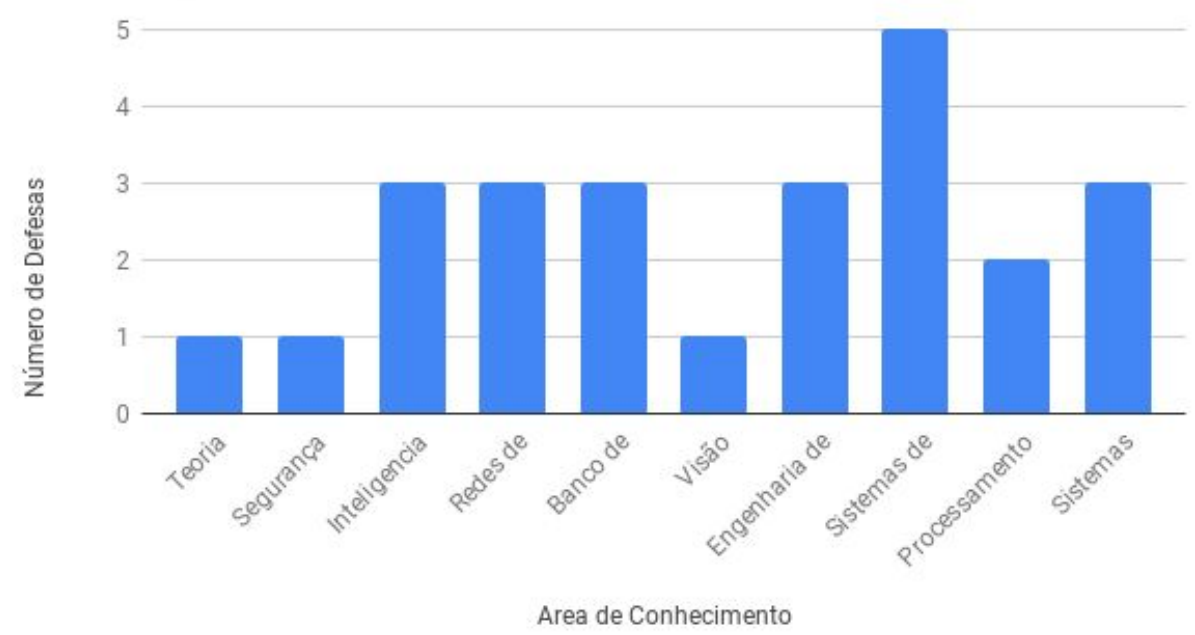

Figure 3. Área de Conhecimento no Mestrado Acadêmico para as Mulheres.

\section{Defesa por Area de Conhecimento}

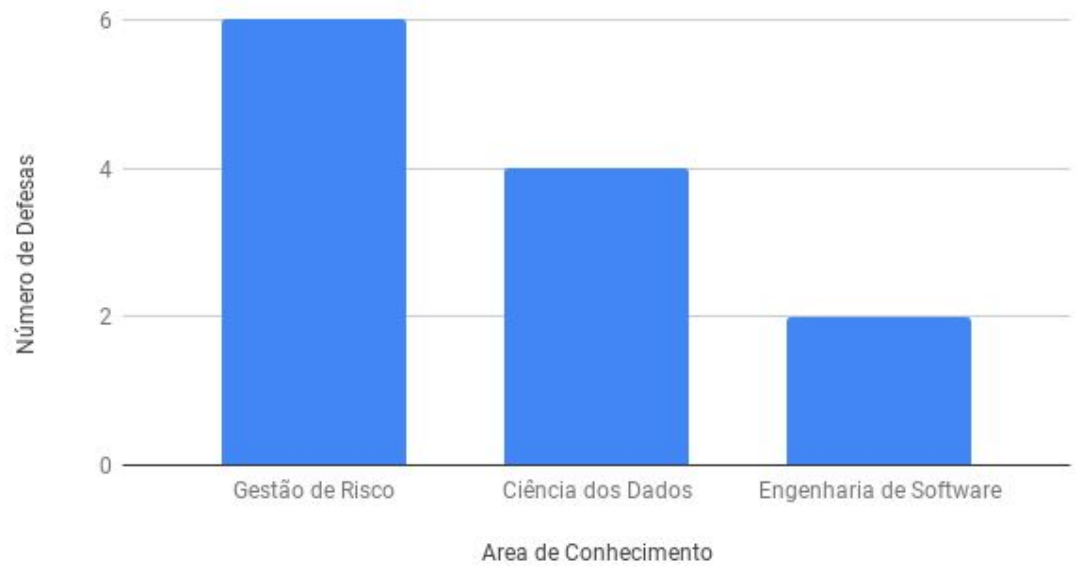

Figure 4. Área de Conhecimento no Mestrado Profissional para as Mulheres.

O primeiro passo para aumentar a inserção feminina na Computação é a formação de mais meninas em graduação na Computação. Neste sentido, o trabalho que os Meninas DIgitais [Maciel, 2018] da Sociedade Brasileira de Computação está realizando, da divulgação da área de Computação para todo o Brasil, é fundamental para que em um futuro próximo seja possível ter mais alunas graduando-se em cursos da área de computação. A UnB tem o projeto Meninas.comp [Holanda, 2016] que é parceiro do Meninas Digitais. 


\section{Conclusão}

Este trabalho apresentou a análise dos dados de pós-graduação do Departamento de Ciência da Computação da Universidade de Brasília no Brasil. Como apresentado, existe uma grande diferença de gênero no mestrado e no doutorado nesta àrea na UnB, com uma inserção de menos de $15 \%$ das mulheres. Assim sendo, ainda tem-se um longo caminho para diminuir a desigualdade de gênero na Ciência da Computação, mas com o incentivo, a divulgação, os projetos motivacionais para inserir mais mulheres na ciência e na computação acredita-se que este cenário deve mudar em um futuro não distante.

Para trabalho futuro, este estudo será expandido para outras universidades do Brasil usando os dados públicos abertos educacionais disponíveis sobre a pós-graduação no portal da CAPES, assim como também uma análise sobre as docentes que atuam nos cursos de pós-graduação na UnB e no Brasil.

\section{Referencias}

Chopra et al. (2019) Gender Differences in Science and Engineering: A Data Mining Approach, Workshop Proceedings of the EDBT/ICDT 20219.

Elsevier (2018) Gender in the Global Research Landscape . Disponível em https://www.elsevier.com/_data/assets/pdf_file/0008/265661/ElsevierGenderReport _final_for-web.pdf

Holanda, M., Dantas, M., Couto, G., Correa, J., Araújo, A., \& Walter, M. (2017). Perfil das Alunas no Departamento de Computação da Universidade de Brasília. In 11 Women in Information Technology (WIT 2017). Porto Alegre: SBC.

Holanda, M. ; Walter, M. E. M. T.; Araujo, A. P. F. (2016). Meninas.comp: computação também é coisa de menina. ParticipAção, Brasília, v. 29, p. 9-19.

Keinan, E., (2017) "A New Frontier: But for Whom? An Analysis of the Micro-Computer and Women's Declining Participation in Computer Science". CMC Senior Teses. 1466.? ? Claremont Colleges.

Maciel, C., Bim, S. A., Figueiredo, K. S. (2018). Digital Girls Program Disseminating Computer Science to Girls in Brazil. In Proceedings GE'18: GE'18:IEEE/ACM 1st International Workshop on Gender Equality in Software Engineering, May 28, 2018, Gothenburg, Sweden.

Maia, M. M. (2016). Limites de gênero e presença feminina nos cursos superiores brasileiros do campo da computação. Cadernos Pagu. n.46, pp. 223-244. ISSN 1809-4449. http://dx.doi.org/10.1590/18094449201600460223.

SBC - Sociedade Brasileira de Computação (2016). Educação Superior Estatística 2016. Disponível em http://www.sbc.org.br/documentos-da-sbc. Acessado em Maio de 2019.

UnB (2019). Repositório de Dissertações e Teses. Disponível em: https://www.bce.unb.br/bibliotecas-digitais/repositorio/teses-e-dissertacoes/

Acessado em Maio de 2019. 